Proceedings of the 2007 Winter Simulation Conference

S. G. Henderson, B. Biller, M.-H. Hsieh, J. Shortle, J. D. Tew, and R. R. Barton, eds.

\title{
AMERICAN OPTION PRICING UNDER STOCHASTIC VOLATILITY: A SIMULATION-BASED APPROACH
}

\author{
Arunachalam Chockalingam \\ School of Industrial Engineering \\ Purdue University \\ West Lafayette, IN 47907, U.S.A.
}

\author{
Kumar Muthuraman \\ McCombs School of Business \\ University of Texas \\ Austin, TX 78712, U.S.A.
}

\begin{abstract}
We consider the problem of pricing American options when the volatility of the underlying asset price is stochastic. No specific stochastic volatility model is assumed for the stochastic process. We propose a simulation-based approach to pricing such options. Iteratively, the method determines the optimal exercise boundary and the associated price function for a general stochastic volatility model. Given an initial guess of the optimal exercise boundary, the Retrospective Approximation (RA) technique is used to calculate the associated value function. Using this function, the exercise boundary is improved and the process repeated till convergence. This method is a simulation based variant of the exercise-policy improvement scheme developed in Chockalingam and Muthuraman (2007). An illustration of the method is provided when using the Heston (1993) model to represent the dynamics of the volatility, together with comparisons against existing methods to validate our numerical results.
\end{abstract}

\section{INTRODUCTION}

Options are contracts that give the holder the right to sell (put) or buy (call) an underlying asset at a pre-determined strike price. A European option allows the holder to exercise the option only on a pre-determined future date known as the expiration date, while an American option allows the holder to exercise the option at any point in time until the expiration date. The problem of pricing options play a central role in financial literature due to their extensive use in financial markets.

Black and Scholes (1973) derive a closed-form solution for the price of a European option. No such solution exists, however, for American options, due to the possibility of early exercise. Researchers have turned to numerical techniques to price American options. The methods proposed by Cox, Ross, and Rubinstein (1979), Kim (1990), Jacka (1991), Carr, Jarrow, and Myneni (1992), Huang, Subrahmanyam, and Yu (1996) and Ju (1998) are some examples. Tilley (1993), Broadie and Glasserman (1997) and Longstaff and Schwartz (2001) are examples of methods that use Monte Carlo (MC) simulation to price an option.

Models under the Black-Scholes setting assume that the volatility of the underlying asset price is constant. Rubinstein (1994) provides empirical evidence using implied volatilities obtained from index options on the S\&P 500 that suggests otherwise. Implied volatilities for in- and out-of-the-money options are higher than at-the-money options. Assuming a constant volatility in the Black-Scholes calculations will therefore lead to options far in-the-money or out-of-themoney being mispriced. Furthermore, Scott (1987) and the references therein give evidence that the volatility of the underlying asset price is not constant and changes over time. Therefore, models assuming that the volatility of the underlying asset price is constant are inadequate for pricing options.

This shortcoming of the Black-Scholes model can be circumvented by letting the volatility of the underlying asset price be stochastic as well. Based on Engle (1982), some researchers model volatility as an ARCH process. Using diffusion processes to model volatility is a more popular choice. Hull and White (1987) assume that the volatility follows a lognormal process and obtain a power series approximation representing the price of the option. A mean-reverting Ornstein-Uhlenbeck (OU) process is used to model volatility in Scott (1987). The solution of the resulting integral of the Black-Scholes equation over the distribution function of the volatility, obtained via MC simulation, is the price of the option. Stein and Stein (1991) also model volatility as a mean-reverting OU process and derive a conditional distribution for the underlying asset price. The option price is obtained by solving an integral expression, using this distribution, representing the expected payoff. Heston (1993) uses a square-root process to describe the dynamics of the volatility and derives a closed-form solution for the price of the option. 


\section{Chockalingam and Muthuraman}

The above-mentioned papers consider the problem of pricing a European option when volatility is stochastic. Relatively little attention, however, has been paid to the problem of pricing an American option when volatility is stochastic. Existing numerical techniques, such as Clarke and Parrott (1999), Oosterlee (2003) and Ikonen and Toivanen (2004), convert the option pricing problem into a linear complementarity problem (LCP), and solve the LCP to obtain the price. Zvan, Forsyth, and Vetzal (1998) discretize the arising Partial Differential Equation (PDE) and solve for the option by using penalty methods. Simulation techniques for pricing high-dimensional American options, such as those proposed by Andersen and Broadie (2004) and Broadie and Glasserman (2004), can be adapted to price options when the volatility is stochastic. The simulation-regression method used by Longstaff and Schwartz (2001) can also be modified to price such options.

Existing methods for solving the American option problem do not solve explicitly for the optimal exercise boundary. The method we propose in this paper is an adaptation of the exercise-policy improvement scheme developed in Chockalingam and Muthuraman (2007), and solves for both the optimal exercise boundary (or policy) and the associated price function simultaneously. Beginning with an initial guess of the exercise policy, the optimal exercise policy and price function are obtained iteratively.

The paper is structured as follows. Section 2 describes the option pricing problem in detail. The method used to obtain the optimal exercise policy is discussed in Section 3. An integral part of obtaining these policies is the determination of the value function associated with a particular policy. We use MC simulation to calculate these value functions. The details of obtaining the value functions are explained in Section 4. We present numerical results in Section 5 and conclude in Section 6.

\section{PROBLEM FORMULATION}

Consider an American put option written on an underlying asset that is traded in a Black-Scholes market with a constant risk-free interest rate $r$. The asset price $X_{t}$ at time $t$ is given by

$$
\begin{aligned}
d X_{t} & =\mu X_{t} d t+\sigma_{t} X_{t} d W_{t} \\
\sigma_{t} & =f\left(Y_{t}\right) \text { and } \\
d Y_{t} & =\theta_{1}\left(Y_{t}\right) d t+\theta_{2}\left(Y_{t}\right) d \hat{Z}_{t}
\end{aligned}
$$

where $\mu$ is the constant mean rate of return and $\sigma_{t}=f\left(Y_{t}\right)$ represents the volatility of the underlying asset price by way of a non-stochastic function $f: \mathbb{R} \rightarrow \mathbb{R}_{+}$. Equation (3) describes the dynamics of the process $Y$, where $\theta_{1}, \theta_{2}$ : $\mathbb{R} \rightarrow \mathbb{R}_{+}$are non-stochastic functions. In Equations (1) and (3), $W_{t}$ and $\hat{Z}_{t}$ are two one-dimensional Wiener processes with constant correlation $\rho \in[-1,1]$, i.e., $d W_{t} d \hat{Z}_{t}=\rho d t$. Hence, $\hat{Z}_{t}$ can be written as a linear combination of $W_{t}$ and an independent Wiener process $Z_{t}$ such that $\hat{Z}_{t}=\rho W_{t}+$ $\sqrt{1-\rho^{2}} Z_{t}$.

Letting $x$ denote the price of the underlying asset at time $t$, a put option with strike price $K$ and maturity time $T$ written on this asset pays $\max \{0, K-x\} \equiv(K-x)^{+}$at any time $t \in[0, T]$. The price of this option at a certain $t, x$ and $y$ is denoted by $p(t, x, y)$, where $y$ represents the value of the process $Y$ at time $t$. Our primary interest lies in determining the price function $p$. Similar to the constant volatility case, the price of the option at a particular $t, x$ and $y$ is given by

$$
p(t, x, y)=\sup _{\tau \in \Pi} \mathbb{E}^{\mathscr{Q}}\left[e^{-r(\tau-t)}\left(K-X_{\tau}\right)^{+}\right]
$$

where $\mathbb{E}^{\mathscr{Q}}$ refers to the expectation taken with respect to the martingale measure $\mathscr{Q}$ and $\Pi$ represents the set of stopping times after $t$. It should be noted that unlike the constant volatility case, $\mathscr{Q}$ is not unique, as the market we have just described is an incomplete one, as a result of volatility of not being a traded asset. There are two sources of randomness, namely $W_{t}$ and $\hat{Z}_{t}$, but only one underlying asset, leading to market incompleteness. The difficulty in pricing American options arises from the fact that the optimal stopping time $\tau^{*}=\sup _{\tau \in \Pi}$ is not known a priori.

Analogous to the constant volatility case, there exists an optimal exercise boundary (or policy) that determines the price of the option. This policy is a surface, $x=b(t, y)$, $b: \mathbb{R}_{+} \times \mathbb{R} \rightarrow \mathbb{R}_{+}$, and dictates how an investor should behave. If $x>b(t, y)$, the investor should hold the option, and exercise it otherwise. Given this policy, determining $\tau^{*}$ becomes trivial, as the optimal stopping time is also defined as

$$
\tau^{*}=\inf _{\tau \in \Pi} X_{\tau} \leq b(\tau, y) .
$$

Essentially, $\tau^{*}$ is the first time that the process describing the underlying asset price strikes the exercise boundary $b$. Thus, given $b$, the optimal stopping time $\tau^{*}$ can be determined, which in turn allows us to determine the option price. The method we propose uses this fact to calculate the price function $p$.

We now list some properties, which will be used in later sections;

$$
\begin{aligned}
b(T, y) & =K, \\
\lim _{x \downarrow b} p_{x}(t, x, y) & =-1 \text { and } \\
p(t, x, y) & \geq(K-x)^{+},
\end{aligned}
$$

where subscripts refer to derivatives. Equation (6) simply states that at time $T$, the value of the boundary is $K$, since no further action is possible after $T$. If the asset price is 


\section{Chockalingam and Muthuraman}

less than $K$, the option should be exercised immediately and be allowed to expire worthless otherwise. Equation (7) describes the behavior of $p$ near $b$. The final equation, Equation (8), states that at all times, the price of an American option should be no less than the value of the immediate payoff, for there would be no reason to hold the option otherwise.

\section{EXERCISE POLICY IMPROVEMENT}

Consider the use of an arbitrary exercise policy $b^{n}$. With such a policy, the investor should hold the option if $x>b^{n}(t, y)$ and hold it otherwise. We call the price function obtained using this policy, $p^{n}$, the value function, so as to avoid confusion with the price function obtained using the optimal exercise policy, $p$. The price function can therefore be viewed as the 'best' value function, i.e., the supremum of all value functions over all possible exercise policies. Now, if $b^{n}(t, y)<b(t, y)$ for all $t$ and $y$, it is shown in Chockalingam and Muthuraman (2007) that $p^{n}$ violates Equations (7) and (8). As a result of this violation, it is possible to pick a new exercise policy $b^{n+1}(t, y)>b(t, y)$ that is associated with a price function $p^{n+1}>p^{n}$. There are several choices of $b^{n+1}$ that assures of policy improvement, but all such choices do not guarantee convergence. It turns out that picking the exercise policy to be the set of furthest points where $p_{x}^{n}(t, x, y)<-1$, i.e.,

$$
b^{n+1}(t, y)=\left\{\sup _{\left.b^{n}(t, y), \infty\right)} x \mid p_{x}^{n}(t, x, y)<-1\right\},
$$

guarantees us of policy improvement and monotone convergence. Policy improvement will not be possible once Equation (7) is satisfied. At this point, the method converges to the optimal exercise policy $b$. The value function associated with this policy is the price function $p$.

As in any numerical method, the infinite state space has to be bounded and discretized. We consider the space $[0, \hat{T}] \times[0, K] \times\left[-\hat{Y}_{1}, \hat{Y}_{2}\right]$, where $\hat{T}, \hat{Y}_{1}, \hat{Y}_{2} \in \mathbb{R}_{+}$are chosen large enough so as to encompass times and volatilities of interest to us. The asset-price axis is bounded at $K$ since $K$ is a guaranteed upper bound for the optimal exercise policy. Now, the time axis, asset-price axis and volatility axis are discretized into $d, c$ and $l$ pieces yielding grid steps $\delta_{t}=\frac{\hat{T}}{d}, \delta_{x}=\frac{K}{c}$ and $\delta_{y}=\frac{\hat{Y}_{1}+\hat{Y}_{2}}{l}$ respectively. For $j=0, \ldots, d, s=0, \ldots, c$ and $r=0, \ldots, l$, the price of the option at node $(j, s, r)$ obtained using the policy $b^{n}$ is denoted by $p^{n}\left(t_{j}, x_{s}, y_{r}\right)$. Note that $t_{d}=\hat{T}, x_{c}=K, y_{0}=-\hat{Y}_{1}$ and $y_{l}=\hat{Y}_{2}$.

To procedure is begun with an initial guess of the exercise policy $b^{0}$. The only condition placed on this guess is that it is less than $b^{0}(t, y)<b(t, y)$. While the perpetual boundary is a guaranteed lower bound for the exercise boundary in the case of constant volatility, no such analogue exists in the stochastic volatility case. Nonetheless, a $b^{0}(t, y)<b(t, y)$ guarantees that $p_{x}^{0}<1$ at the boundary $b_{0}$. This test can be used to confirm that $b^{0}(t, y)<b(t, y)$, or else a restart with another guess can be made. Now, using $b^{0}, p^{0}\left(t_{d-1}, x_{s}, y_{r}\right)$ is obtained for all $s$ and $r$. The MC simulation used to obtain this is discussed in the next section. For each $s$ and $r$, the first derivative, $p_{x}^{0}$ is then calculated, and the new policy, $b^{1}\left(t_{d-1}, y_{r}\right)$, chosen according to Equation (9) for all $r$. The new value function, $p^{1}\left(t_{d-1}, x_{s}, y_{r}\right)$, is determined and the process repeated until convergence to within a preset tolerance. The method steps back in time to $t_{d-2}$, and the process starts anew, with $b^{0}$ again. This is done repeatedly till time $t_{0}$, at which point calculations terminate, leaving us with $b$ and $p$. The convergence of this procedure to the option price, is provided in Chockalingam and Muthuraman (2007) for the Heston (1993), Hull and White (1987), Stein and Stein (1991) and Scott (1987) models.

\section{PRICE FUNCTION DETERMINATION}

Given an exercise policy $b^{n}$, the associated value function $p^{n}$ has to be determined so that Equation (9) can be used to obtain a new policy $b^{n+1}$. We use the Retrospective Approximation (RA) technique proposed by Chen and Schmeiser (2001) to determine these value functions.

Consider the policy $b^{n}$, i.e., the exercise policy used during iteration $n$. At node $\left(t_{j}, x_{s}, y_{r}\right)$, if $x_{s} \leq b^{n}\left(t_{j}, y_{r}\right)$, we have $p^{n}\left(t_{j}, x_{s}, y_{r}\right)=\left(K-x_{s}\right)^{+}$. If, however, $x_{s}>b^{n}\left(t_{j}, y_{r}\right)$, the value of the option at this node is determined by $\mathrm{MC}$ simulation. First, the space $\left[t_{j}, \hat{T}\right]$ is further discretized into $k$ uniform pieces, where $k$ is a fixed constant determined beforehand. Each of these times is denoted by $t_{j}^{h}$, where $h=0, \ldots, k$, with $t_{j}^{0}=t_{j}, t_{j}^{k}=\hat{T}$ and a grid step of $\delta_{h}=\frac{\hat{T}-t_{j}}{k}$. Intermediate values of the exercise policy from $t_{j}$ to $\hat{T}$ have to be obtained by interpolation for all $r$. Now, $m_{n}$ sample paths of the asset price have to be generated, starting from the node $\left(t_{j}, x_{s}, y_{r}\right)$. The RA technique is used to control sample path generation. The number of sample paths to be used during iteration $n$ depends on the number of sample paths used during iteration $n-1$. During earlier iterations, lesser accuracy is required. As the iteration number increases, however, greater accuracy is needed, so as to allow the method to converge to the optimal exercise policy. Thus, the number of sample paths used during each iteration grow according to a pre-specified rule, namely $m_{n}=a m_{n-1}$, where $a>1$ is also a fixed constant determined beforehand.

Sample-path generation is dependent on the specific stochastic volatility model chosen. For illustrative purposes, we consider the Heston (1993) model. Under this model, 
Equations (1) - (3) become

$$
\begin{aligned}
d X_{t} & =r X_{t} d t+\sigma_{t} X_{t} d W_{t} \\
\sigma_{t} & =\sqrt{Y_{t}} \text { and } \\
d Y_{t} & =\left(\kappa\left(m^{\prime}-Y_{t}\right)-\Lambda Y_{t}\right) d t+v \sqrt{Y_{t}} d \hat{Z}_{t}
\end{aligned}
$$

where $\kappa$ refers to the rate at which the process $Y$ reverts to its mean value of $m^{\prime}$ and $v$ refers to the volatility of the process, with $\kappa, m^{\prime}, v \in \mathbb{R}_{+}$. In Equation (12), the $\Lambda$ term refers to the volatility risk premium which arises as a result of market incompleteness. Thus, in Equation (10), $\mu$ is replaced by $r$. Recall from Section 2 that the probability mesure $\mathscr{Q}$ is not unique. In fact, choosing $\mathscr{Q}$ is equivalent to choosing $\Lambda$. For simplicity, we assume that $\Lambda \in \mathbb{R}$ is a constant.

We now explain how a single observation of the asset price is obtained. Generating a sample path is a straightforward extension. By Equations (10) - (12), we have

$$
\begin{aligned}
X_{t_{j}^{h}}= & X_{t_{j}^{h-1}}\left(1+r \delta_{h}+\sqrt{Y_{t_{j}^{h}} \delta_{h}} N\right. \text { and } \\
Y_{t_{j}^{h}}= & Y_{t_{j}^{h-1}}\left(1-(\kappa-\Lambda) \delta_{h}\right) \\
& +\kappa m^{\prime} \delta_{h}+v \sqrt{Y_{t_{j}^{h-1}}} \hat{Z}
\end{aligned}
$$

where $N$ and $\hat{Z}$ are two $N(0,1)$ random variables with correlation $\rho$. To obtain $\hat{Z}$, first, two independent $N(0,1)$ random variables, $N$ and $Z$, are generated. Then, $\hat{Z}=\rho N+\sqrt{1-\rho^{2}} Z$. Now, substituting values into Equation (14) yields $Y_{t_{j}^{h}}$, which is in turn used in Equation (13) with $N$ to yield $X_{t_{j}^{h}}$. Repeating this process provides a complete sample path from $t_{j}$ to $\hat{T}$. Note that in Equation (13), $X_{t_{j}^{0}}=x_{s}$ and in Equation (14), $Y_{t_{j}^{0}}=y_{r}$, when the sample path is started from node $\left(t_{j}, x_{s}, y_{r}\right)$.

Now say $m_{n}$ sample paths have been generated, indexed by $i$, such that $i=1, \ldots, m_{n}$. For each path $i$, the first time, $\tau_{i}$, that the path strikes the policy $b^{n}$ between $t_{j}$ and $\hat{T}$, along with the value of the policy at that time, $b_{i}^{n}$, is recorded. The value of the option along that path is then $p^{n, i}\left(t_{j}, x_{s}, y_{r}\right)=e^{-r\left(\tau_{i}-t_{j}\right)}\left(K-b_{i}^{n}\right)$. Finally, when the value of the option along all $m_{n}$ paths has been calculated, the value of the option at the node $\left(t_{j}, x_{s}, y_{r}\right)$ is given by $p^{n}\left(t_{j}, x_{s}, y_{r}\right)=\sum_{i=1}^{m_{n}} p^{n, i}\left(t_{j}, x_{s}, y_{r}\right) / m_{n}$.

\section{AN ILLUSTRATIVE EXAMPLE}

We provide an numerical example to demonstrate the validity of the method for the stochastic volatility model proposed by Heston (1993). We compare results obtained using the simulation method against results obtained using the finite difference method proposed in Chockalingam and Muthuraman (2007). Table 1 describes the model parameters used for the example. Tables 2 and 3 list the algorithm parameters used for the simulation-based method and the finite-difference method.

Table 1: Model parameters

\begin{tabular}{|c|c|}
\hline Model Parameters & Values \\
\hline$K$ & 10 \\
\hline$T$ & 0.25 years \\
\hline$r$ & 0.1 \\
\hline$\kappa$ & 5 \\
\hline$m^{\prime}$ & 0.16 \\
\hline$v$ & 0.9 \\
\hline$\rho$ & 0.1 \\
\hline$\Lambda$ & 0 \\
\hline
\end{tabular}

Table 2: Simulation (Sim) parameters

\begin{tabular}{|c|c|}
\hline Simulation Parameters & Values \\
\hline$\hat{Y}_{1}$ & 0 \\
\hline$\hat{Y}_{2}$ & 1 \\
\hline$\hat{T}$ & 0.25 \\
\hline$c$ & 80 \\
\hline$l$ & 32 \\
\hline$d$ & 16 \\
\hline$k$ & 250 \\
\hline$a$ & 1.5 \\
\hline$m_{0}$ & 100 \\
\hline$\varepsilon$ & 0.05 \\
\hline
\end{tabular}

Table 3: Finite difference (FD) parameters

\begin{tabular}{|c|c|}
\hline FD parameters & Values \\
\hline$\hat{X}$ & 20 \\
\hline$\hat{Y}_{1}$ & 0 \\
\hline$\hat{Y}_{2}$ & 1 \\
\hline$\hat{T}$ & 0.25 \\
\hline$c$ & 80 \\
\hline$l$ & 32 \\
\hline$d$ & 16 \\
\hline
\end{tabular}

The algorithm needs to be given a guess of the boundary before computations can take place. We set the initial guess of the boundary to be $b^{0}(t, y)=1$. Table 4 provides a comparison of option prices obtained using the simulationbased method and the finite-difference method for 5 different asset prices as 5 different volatilities.

As the table demonstrates, option prices obtained using the simulation-based method are comparable to option prices obtained using the finite-difference scheme. As with any simulation technique, greater accuracy can be obtained by increasing the number of sample paths used, or in this case, by either increasing the initial number of sample paths or the retrospective approximation parameter. 
Table 4: Comparison of option prices

\begin{tabular}{|c||c|c|c|c|c|}
\hline \multicolumn{1}{|c||}{} & \multicolumn{5}{c|}{ Stock price, $x$} \\
\cline { 2 - 6 } \multicolumn{1}{|c||}{} & 8 & 9 & 10 & 11 & 12 \\
\hline \multicolumn{1}{|c||}{$\mathrm{y}=0.25$} & \multicolumn{5}{|c|}{} \\
\hline \multicolumn{1}{|c||}{ Sim } & 2.127 & 1.347 & 0.796 & 0.446 & 0.235 \\
\hline \multicolumn{1}{|c||}{ FD } & 2.074 & 1.325 & 0.785 & 0.440 & 0.238 \\
\hline \multicolumn{1}{|c||}{$\mathrm{y}=0.375$} & \multicolumn{5}{|c|}{} \\
\hline \multicolumn{1}{|c||}{ Sim } & 2.175 & 1.448 & 0.928 & 0.573 & 0.343 \\
\hline \multicolumn{1}{|c||}{ FD } & 2.149 & 1.448 & 0.928 & 0.572 & 0.344 \\
\hline $\mathrm{y}=0.5$ & \multicolumn{5}{|c|}{} \\
\hline \multicolumn{1}{|c||}{ Sim } & 2.229 & 1.548 & 1.047 & 0.687 & 0.446 \\
\hline \multicolumn{1}{|c||}{ FD } & 2.225 & 1.558 & 1.052 & 0.691 & 0.446 \\
\hline $\mathrm{y}=0.625$ & \multicolumn{5}{|c|}{} \\
\hline \multicolumn{1}{|c||}{ Sim } & 2.288 & 1.638 & 1.150 & 0.787 & 0.543 \\
\hline \multicolumn{1}{|c||}{ FD } & 2.299 & 1.657 & 1.163 & 0.800 & 0.543 \\
\hline $\mathrm{y}=0.75$ & \multicolumn{5}{|c|}{} \\
\hline Sim & 2.339 & 1.724 & 1.243 & 0.883 & 0.630 \\
\hline FD & 2.367 & 1.747 & 1.262 & 0.898 & 0.633 \\
\hline
\end{tabular}

\section{CONCLUSION}

When the volatility of an asset is assumed to be constant, pricing an American option written on that asset is not trivial. The volatility of an asset, however, is not constant. As such, we have considered the problem of pricing an American option written on an underlying asset whose volatility is stochastic. A simulation-based method similar in spirit to the finite-difference scheme proposed in Chockalingam and Muthuraman (2007) is used to calculate both the optimal exercise policy and the price function. An example was provided to illustrate the validity of results obtained using this method.

It is clear that there is further scope for improvement. The effects of the simulation parameters, such as the rate at which the number of sample paths should increase, have not been studied. A better of understanding of these parameters in this context could lead to rules being devised according to which these parameters should be chosen. The method we have described pertains to the one asset case. Another area for development would be to extend the algorithm to price multidimensional options, i.e., options written on multiple underlying assets.

\section{REFERENCES}

Andersen, L., and M. Broadie. 2004. Primal-Dual Simulation Algorithm for Pricing Multidimensional American Options. Management Science 50 (9): 1222-1234.

Black, F., and M. Scholes. 1973. The Pricing of Options and Corporate Liabilities. J. Political Economy 81 (3): 637-654.
Broadie, M., and P. Glasserman. 1997. Pricing AmericanStyle Securities by Simulation. J. Economic Dynamics and Control 21:1323-1352.

Broadie, M., and P. Glasserman. 2004. A Stochastic Mesh Method for Pricing High-Dimensional American Options. J. of Computational Finance 7 (4): 35-72.

Carr, P., R. Jarrow, and R. Myneni. 1992. Alternative Characterizations of American Put Options. Mathematical Finance 2:87-106.

Chen, H., and B. Schmeiser. 2001. Stochastic Root Finding via Retrospective Approximation. IIE Transactions 33 (3): 259-275.

Chockalingam, A., and K. Muthuraman. 2007. American Options under Stochastic Volatility. Working paper.

Clarke, N., and K. Parrott. 1999. Multigrid for American Option Pricing with Stochastic Volatility. Applied Mathematical Finance 6:177-195.

Cox, J. C., S. A. Ross, and M. Rubinstein. 1979. Option Pricing: A Simplified Approach. J. of Financial Economics 7:229-263.

Engle, R. F. 1982. Autoregressive Conditional Heteroskedasticity with Estimates of the Variance of U.K. Inflation. Econometrica 50:987-1008.

Heston, S. L. 1993. A Closed-form Solution for Options with Stochastic Volatility and Applications to Bond and Currency Options. Review of Financial Studies 6 (2): 327-343.

Huang, J., M. Subrahmanyam, and G. Yu. 1996. Pricing and Hedging American Options: A Recursive Integration Method. Review of Financial Studies 9:277-300.

Hull, J., and A. White. 1987. The Pricing of Options on Assets with Stochastic Volatilities. J. of Finance 42 (2): 281-300.

Ikonen, S., and J. Toivanen. 2004. Operator Splitting Methods for Pricing American Options with Stochastic Volatility. Technical report, Department of Mathematical Information Technology, University of Jyväskylä.

Jacka, S. D. 1991. Optimal Stopping and the American Put. Mathematical Finance 1:1-14.

Ju, N. 1998. Pricing an American Option by Approximating its Early Exercise Boundary as a Multipiece Exponential Function. Review of Financial Studies 11 (3): 627-646.

Kim, I. J. 1990. The Analytic Valuation of American Options. Review of Financial Studies 3:547-572.

Longstaff, F. A., and E. S. Schwartz. 2001. Valuing American Options by Simulation: Simple Least-Squares Approach. Review of Financial Studies 14:113-147.

Oosterlee, C. W. 2003. On Multigrid for Linear Complementarity Problems with Application to American-style Options. Electronic Transactions on Numerical Analysis 15:165-185.

Rubinstein, M. 1994. Implied Binomial Trees. J. of Finance 49 (3): 771-818. 
Scott, L. O. 1987. Option Pricing when the Variance Changes Randomly: Theory, Estimation, and an Application. $J$. of Financial and Quantitative Analysis 22 (4): 419-438.

Stein, E. M., and J. C. Stein. 1991. Stock Price Distributions with Stochastic Volatility: An Analytic Approach. Review of Financial Studies 4 (4): 727-752.

Tilley, J. A. 1993. Valuing American Options in a Path Simulation Model. Transactions of the Society of Actuaries 45:83-104.

Zvan, R., P. A. Forsyth, and K. R. Vetzal. 1998. Penalty Methods for American Options with Stochastic Volatility. J. Comput. Appl. Math. 91 (2): 199-218.

\section{AUTHOR BIOGRAPHIES}

ARUNACHALAM CHOCKALINGAM is a Ph.D. student in the School of Industrial Engineering at Purdue University, where he received his M.S. in 2006. His interests broadly lie in the application of simulation techniques to problems in financial engineering. His e-mail address is <arunachalamepurdue.edu>.

KUMAR MUTHURAMAN is an assistant professor in the McCombs School of Business at the University of Texas at Austin. He received his Ph.D from Stanford University in 2003. His interests lie in mathematical finance and stochastic control. His e-mail address is $<$ kumarepurdue. edu $>$. 